\title{
Fuzzy Object Oriented Database versus FRDB for Uncertainty Management
}

\author{
Sonia \\ M.Tech in Computer Science and Engg. \\ Maharishi Dayanand University \\ Rohtak, India
}

\author{
Subita Kumari \\ Lecturer in Computer Engg. \\ Govt. Polytechnic Sanghi \\ Rohtak, India
}

\begin{abstract}
It is impossible to capture all of the semantics of real world information and to model it in a perfect way. Uncertain information being lacking information, individual interpretation of knowledge is taken into account. Most widely used relational model faced much difficulty to handle complex data requirements. The size of databases and their changing composition such as graphics, video and sound, as well as numbers and text invited a reorganization of existing information systems. Object oriented database draw their strength from graphical user interfaces, advanced data management capabilities and powerful modeling techniques. So in order to deal with inexactness fuzzy techniques have been extensively integrated with more advanced object oriented database system which is capable to represent and manipulate the complex objects as well as complicated and uncertain relationship exiting among them. In this paper uncertainty using Fuzzy Object Oriented Database and Fuzzy Relational Data Base is illustrated.
\end{abstract}

\section{KEYWORDS}

FOOD (Fuzzy Object Oriented Databases), FRDB (Fuzzy relational databases), OODBMS (Object Oriented Database Management System)

\section{INTRODUCTION}

Object oriented databases are considered better than relational databases, due to increasing demand of new approaches to deal with complex data, complex relationship exiting among such data and large data intensive applications. Object oriented databases are much suitable for modern database applications, like CAD/CAM (Computer Aided Design/Computer Aided Manufacturing), CASE(Computer Aided Software Engineering), GIS (Geographical Information Systems), Spatial Databases, Office Automation; Knowledge based Systems, Hardware and Software Design, Network Management, Multimedia databases, VLSI (Very Large Scale Integrated) Design. In these applications, several types of information inexactness exist. Such incomplete and ill-defined information has been accepted, represented and manipulated with a certainty measure of acceptance using fuzzy techniques. So FOOD deal with different fuzzy concepts, like =almost all, =majority, =approximately, which include uncertainty. Complex object Structures can be represented well without fragmentation of aggregate data and complex relationship among attributes. Fuzzy object oriented database shows lack of formal semantics and algebra for manipulation and representation of knowledge as well as the inexact information data/information. Fuzzy relational database don't use the concept of reusability, but reusability of classes allows for faster development and easier maintenance of the database and its application in FOOD.

\section{DATABASES}

Database is a repository for heterogeneous but interrelated pieces of information. It is a structured collection of data, which are intentionally brought together, made persistent and suited for querying. We can derive semantically meaningful data by querying the database. If database comprises knowledge, which is represented in the form of logical rules, is called as knowledge base. Data model are being used to model the descriptive part of data, which help to understands records, fields and linear file of homogenous records.

\subsection{Inexactness in Information}

The knowledge about the real world is deficient and as a consequence, its modeling and its representation is imperfect. It is impossible to capture all of the semantics of real world information. Uncertainty can exist about the data, about the meaning of data, taking into account that data eventually can draw their meaning from the comparison with other data through their mutual or supposed proximity or similarity. Thus inexactness in the information could be of following forms:

- Uncertainty (when it is not possible to determine whether an assertion in the model is true or false)

- Imprecision (when the information available in the model is not as specific as it should be)

- Vagueness (when the model includes elements that are inherently vague)

- Inconsistency (when the model contains two or more assertions that cannot be true at the same time)

- Ambiguity (when some elements of the model lack complete semantics, leading to several possible interpretations).

\subsection{Fuzzy Relational Databases}

It is an extension of the relational database. This extension introduces fuzzy predicates under shapes of linguistic expressions that, at the time of a flexible querying, permits to have a range of answers (each one with a membership degree) in order to offer to the user all intermediate variations between the completely satisfactory answers and those completely dissatisfactory. So Fuzzy Relational Data Base Management System (FRDBMS) uses flexible queries that allows the user to have a range of answers (each one with its membership degree) offering all intermediate variations between the completely satisfactory and dissatisfactory values. 
FRDBMS is considered first of all a database. It must be made up of core of database which permits to execute the classical operations of the DBMS and to store, manipulate the fuzzy attributes. We can add flexibility in database in many forms.
The OODB is based on the concepts derived from OOPL. FOOD model is used as logical data model. In FOOD model, regarding the representation of imprecise information, uncertainty is handled at three levels: attribute level,

\section{TABLE I. Data types used in the GEFRED model}

1. A single number (e.g., Age $=20$, represented by the possibility of distribution $1 / 20$ ).

2. A single scalar (e.g., Behavior=bad, represented by the possibility of distribution $1 / \mathrm{bad}$ ).

3. A set of mutually exclusive possible numeric assignations (e.g., Age $=\{25,26\}$, represented by $\{1 / 25,1 / 26\}$ ).

4. A set of mutually exclusive possible scalar assignations (e.g., Behavior $=\{\mathrm{Bad}, \mathrm{Good}\}$, represented by $\{1=\mathrm{Bad}, 1=\mathrm{Good}\})$.

5. A possibility distribution in a numeric domain (e.g. Age $=\{0.4 / 19,1.0 / 20,0.8 / 21\}$, fuzzy numbers or linguistic labels).

6. A possibility distribution in a scalar domain (e.g., Behavior $=\{0.6 / \mathrm{Bad}, 1.0 / \mathrm{Regular}\}$ ).

7. A real number belonging to $[0,1]$, referring to the degree of matching (e.g., Quality=0.9).

8. An Undefined value with possibility distribution Undefined $=\{0 / \mathrm{u}: \mathrm{u} \in U\}$ on domain $U$, considered

9. An Unknown value with possibility distribution Unknown $=\{1 / \mathrm{u}: \mathrm{u} \in \mathrm{U}\}$ on domain $\mathrm{U}$, considered.

10. A NULL value given by NULL $=\{1 /$ Undefined, $1 /$ Unknown $\}$.

The

simplest one is to add a fuzzy membership degree to each record (an attribute in range $[0,1]$ ). Fuzzy query being a querying tool, it improves the meaning of the query as well as extracts additional valuable information. Linguistic expressions and degrees of truth are being used to select the wanted scenario for the user. These linguistic expressions have logical meaning for user and define a data selection process in the natural language.

\subsection{Fuzzy Object Oriented Database}

Object-oriented database have a significant advantage over their predecessors, it uses more complex data models thus provide higher level of data abstraction and data independence. They are suited for more sophisticated applications; whole software environment can be built on the same principles, using the same programming tools. Transaction management, authorization control, portability, interoperability etc. all of them can be realized. Object-oriented database programming is less cumbersome so greatly improves the productivity of the programmer.

FOOD uses objects. Object stand for real world or abstract entities and consist of encapsulated data structures, possibly of great complexity, having behavioral capacities. Based on their properties of structure and behavior, objects belong to classes, which can be related to each other in subclass, superclass hierarchies, thus supporting inheritance. The combined fuzzy and uncertain object-oriented databases offer the power of object-orientation and the availability of a richer semantic expressiveness. The uncertainty in classification and in the inheritance hierarchies in class/subclass relationships, as a consequence of the allowed imprecision in data is modeled by using fuzzy set theory.

\section{UNCERTAINTY OF FUZZY OBJECT ORIENTED DATABASE AND FUZZY RELATIONAL DATABASE}

In fuzzy relational databases the uncertainty is related to tuples or attributes as there is no concept of classes and objects in FRDB. The fuzzy relational databases capture impreciseness and incompleteness in data in a better way. The data types used in FRDB are explained by GEFRED model which are given in Table I. [1] [4] class/superclass level and object/class level. But in FRDB there is no concept of object/class, class/superclass level. [3]

\subsection{Attribute level uncertainty}

FOOD deals with 3 types of uncertainty at the attribute level.

- The first type being incomplete type when the value of the attribute is specified as a range value (e.g. 100200). This type is called "incompleteness."

- The second type of uncertainty occurs when the value of the attribute is unknown, does not exist or there is no information on whether a value exists or not. This type of uncertainty is called "null".

- The third type of uncertainty occurs when the value of the attribute is vaguely specified. This type of uncertainty is called "fuzzy".

FOOD allows definition of relevance for fuzzy attributes, which is a real number between 0 and 1 reflecting the importance of the range definition of that fuzzy attribute in defining the boundaries of its class. Range and relevance are used to find the membership degree of an object to its class, and the membership degree of a class to its superclass. They are the same for each instance of a class.

\subsection{Object/Class Level Uncertainty}

Uncertainty at the object/class level refers to the existence of a partial membership of an object to its class. In FOOD model, the boundaries of a class might be uncertain since it has fuzzy attributes. Range of a fuzzy attribute indicates ideal values for that attribute. Since a fuzzy attribute may take any value from its domain regardless of its range definition, some objects are full members of their classes with a membership degree of 1 whereas some objects are member of their classes with a membership degree changing between 0 and 1 . The values of fuzzy attributes of an object determine the membership degree of that object to its class. The closer the value of fuzzy attributes of an object to range definitions, the higher the object membership degree. Relevance of the fuzzy attributes and the similarity between the fuzzy attributes' values and their range definitions determine the membership degree of an object to its class.

Fuzziness in object oriented database is shown in Table II. [9] 
TABLE II. Fuzziness in object-oriented databases

\begin{tabular}{|l|l|l|l|l|l|}
\hline \multicolumn{1}{|c|}{ Focus } & \multicolumn{1}{|c|}{ Fuzziness in object } & $\begin{array}{c}\text { Fuzziness in } \\
\text { class }\end{array}$ & \multicolumn{1}{c|}{$\begin{array}{c}\text { Fuzziness in object- } \\
\text { class }\end{array}$} & \multicolumn{1}{c|}{$\begin{array}{c}\text { Fuzziness in class- } \\
\text { subclass }\end{array}$} & Operation \\
\hline $\begin{array}{l}\text { Imprecision } \\
\text { data } \\
\text { managemen } \\
\text { t }\end{array}$ & Attribute type & Yes & No & Explicit & $\begin{array}{l}\text { Graph } \\
\text { operation }\end{array}$ \\
\hline $\begin{array}{l}\text { Uncertainty } \\
\text { in hierarchy }\end{array}$ & Range of attribute value & Yes & membership degree & $\begin{array}{l}\text { Weak and strong class } \\
\text { hierarchy }\end{array}$ & No \\
\hline $\begin{array}{l}\text { Semantic } \\
\text { data model }\end{array}$ & Imprecision in attribute & Imprecision & Fuzzy similarity & Fuzzy similarity & Fuzzy rules \\
\hline $\begin{array}{l}\text { Fuzzy } \\
\text { classificatio } \\
n\end{array}$ & Uncertainty in attribute & Yes & Fuzzy predicate & Fuzzy predicate & Fuzzy rules \\
\hline $\begin{array}{l}\text { Fuzzy } \\
\text { intelligent } \\
\text { architecture }\end{array}$ & $\begin{array}{l}\text { Possibility distribution } \\
\text { fuzzy values }\end{array}$ & Yes & Fuzzy inclusion & Fuzzy implication & Fuzzy rules \\
\hline $\begin{array}{l}\text { Modeling } \\
\text { fuzziness } \\
\text { and } \\
\text { uncertainty }\end{array}$ & Linguistic attribute & Uncertain & Uncertain & Uncertain & No \\
\hline
\end{tabular}

\subsection{Class/Subclass Level Uncertainty}

Uncertainty at the class/subclass level refers to the existence of a partial membership of a class to its superclass. This type of uncertainty indicates that the fuzziness occurs at the class inheritance hierarchy since a class hierarchy might not be constructed precisely in some cases.

\section{COMPARISON OF FOOD AND FRDB}

\subsection{Object, Entity set, Tuple and Table}

The concept of object in object oriented database is beyond the concept of entity and tuple in relational database. This has additional characteristics such as behavior, inheritance and encapsulation. The E-R approach requires the use of two different entities to model a single real world entity. In contrast, the FOOD model's object is directly modeled as an object into the object space or object schema.

Class is a more powerful concept that allows not only the description of the data structure but also the concept and implementation of abstract data types in the object oriented database. The ADT is a very powerful modeling tool because it allows the end user to create new data types and use them as any other base data types that accompanies a database. The ADT thus yields an increase in the semantic content of the objects being modeled.

Data types of fuzzy attributes are shown below: [1] [4]

- Fuzzy Attributes Type 1: The attributes with "precise data", crisp (with no imprecision). But these may have linguistic labels, which allow making the query conditions more flexible.
- $\quad$ Fuzzy Attributes Type 2: These attributes allow both crisp and fuzzy data, in the form of possibility distributions over fuzzy sets. It is an extension of the Type1 which allow the storage of imprecise information.

- Fuzzy Attributes Type 3: They are attributes over "data of discreet non-ordered dominion with analogy". In which the relationship indicates to what extent each pair of labels be similar to each other.

- Fuzzy Attributes Type 4: These attributes are like Type 3 attributes but does not show similarity relationship between labels.

\subsection{Data Model Representation}

In relational database, connections between two relations are represented by foreign key attribute in one relation that references the primary key of another relation. Individual tuples having same values in foreign and primary key attribute are logically related. Relational model uses logical references.

In object oriented database, relationships are represented by references via the object identifier (OID). These are similar to foreign keys but at place of user-defined attributes internal system identifiers are being used. The Object Oriented model supports complex object structures, specification of methods and inheritance mechanism by using tuple, list, set and other constructors. It helps in creation of new class definitions from existing ones. [2]

\subsection{Storage Structures}

In relational database, each relation is implemented as a separate file and in each file tuples are stored without any order if storage structure is not specified. 


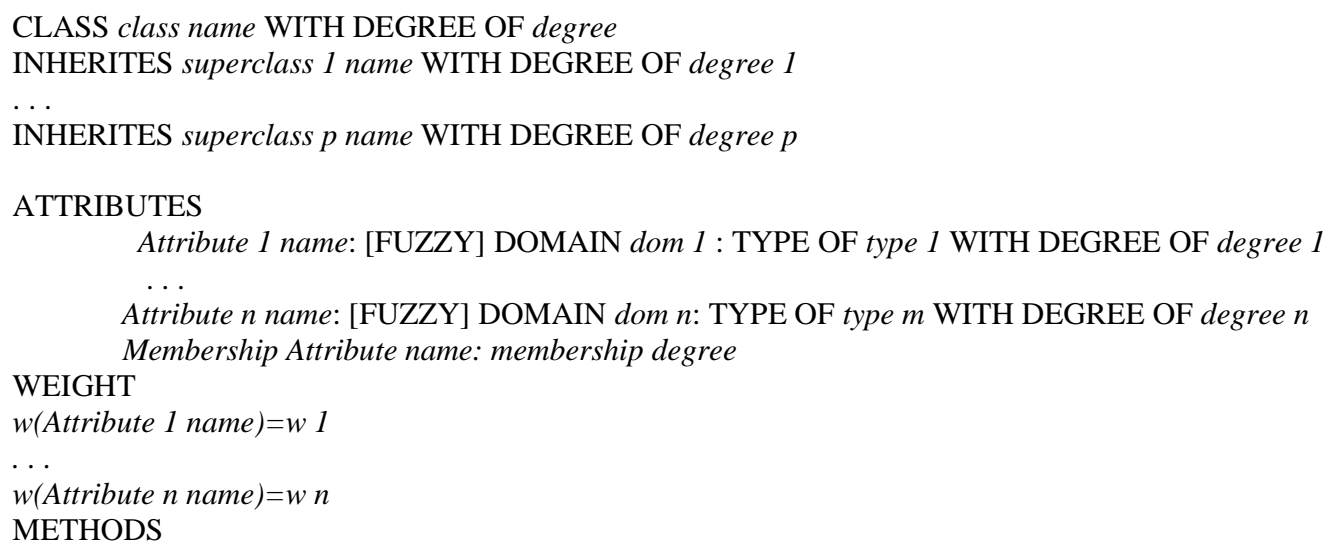

In FRDB the user can dynamically specify a single primary or clustering index and any number of secondary indexes on each file. In some RDBMSs user can access related records from more than one relation by placing a record from one relation followed by the related records from another relation, thus retrieving the related records in most efficient way.

Object oriented systems provide persistent storage for complexstructured objects and employ indexing techniques to locate disk pages. We can reconstruct the object structure after copying the disk pages that contains object into system buffers. The objects are often stored as byte strings. [8]

\subsection{Integrity Constraints}

Relational model has foreign keys, primary keys so entity integrity and referential integrity are used. But in Object Oriented systems these constraints vary from system to system. Some OODB supports inverse relationship mechanism which provides some declarative constraints.

\subsection{Data Manipulation Languages}

There are non-procedural query languages for relational systems such as SQL, QUEL, and QBE, which are based on relational calculus. In OODB, Object Data Management Group (ODMG) has proposed OQL as standard query language. In these systems, the DML is incorporated into some programming language, such as $\mathrm{C}++$, which makes the structures of both stored persistent objects and programming language transient objects compatible to each other.

FOQL is extension of ODMG-OQL, which can deal with fuzzy object model and fuzzy data.

\subsection{Relationships}

The relationships in an FOOD can be of two types: interclass references or class hierarchy inheritance. The E-R and the relational models use a value-based relationship approach. Using a value-based approach means that a relationship among entities is established through a common value in one or several of the entity attributes.

In contrast FOOD uses the object ID, which is identity-based, to establish relationships among objects and such relationships are independent of the state of the object. [3]

\subsection{Example}

The example given in Fig. 1 shows the new constructors added to the UML model to represent uncertainty at object/class and class/subclass levels. The class EndangeredSpecies is the superclass, the classes Butterfly, EndangeredBear, and Wolf are the subclasses. The inheritance relationship in this example is fuzzy; therefore, the classes Butterfly, EndangeredBear and Wolf are the subclasses of EndangeredSpecies with a membership degree, which may change between 0 and 1. [9] The class EndangeredBear has " $|\mathrm{U}|$ " notation indicating that it is also uncertain at the object/class level. The instances of EndangeredBear class are the members of the class with a membership degree, which may change between zero and one. As it is represented in Fig. 1, there are three instances of the class EndangeredBear: KoalaBear, PolarBear and PandaBear. KoalaBear is an instance of the EndangeredBear class with a membership degree of 0.5 , PolarBear is an instance with a membership degree of 0.8 and PandaBear is an instance with a membership degree of 1 , which means it has a full membership to the EndangeredBear class. 
Fig. 1. Object/Class and Class/Subclass Level Uncertaintie

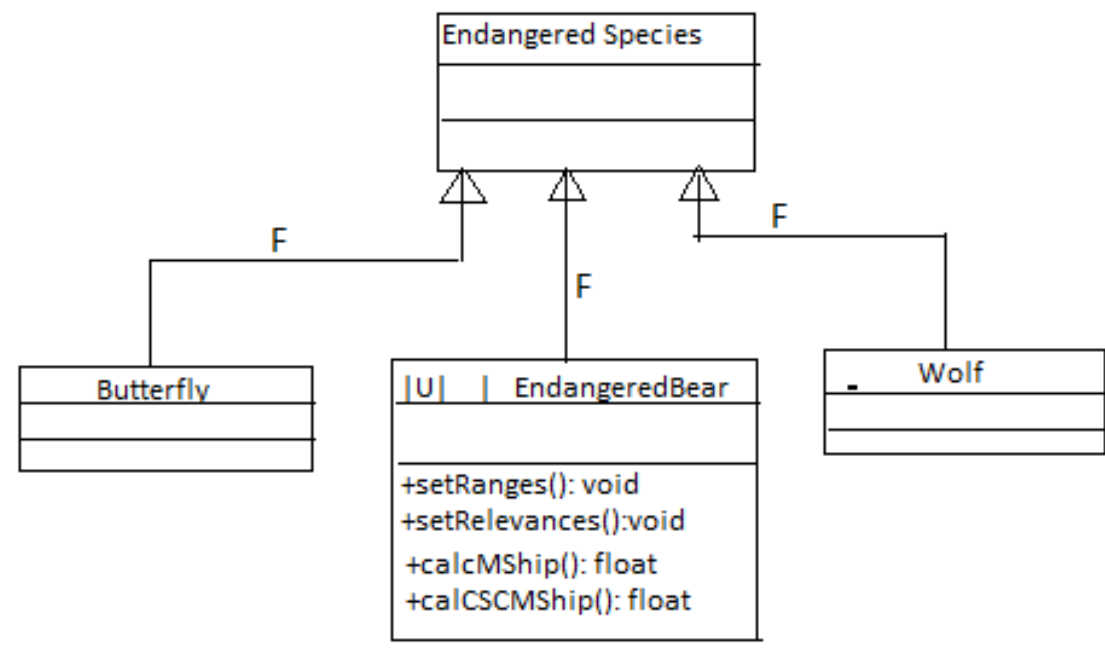

\begin{tabular}{|l|}
\hline KoalaBear: EndangeredBear \\
\hline ObjectMShip: float $=0.5$ \\
\hline
\end{tabular}

PolarBear:EndangeredBear

ObjectMShip: float $=0.8$

PandaBear:EndangeredBear ObjectMShip: float= 1

Fig. 2. Fuzzy Attributes, fuzzy entities and relationships in FRDB

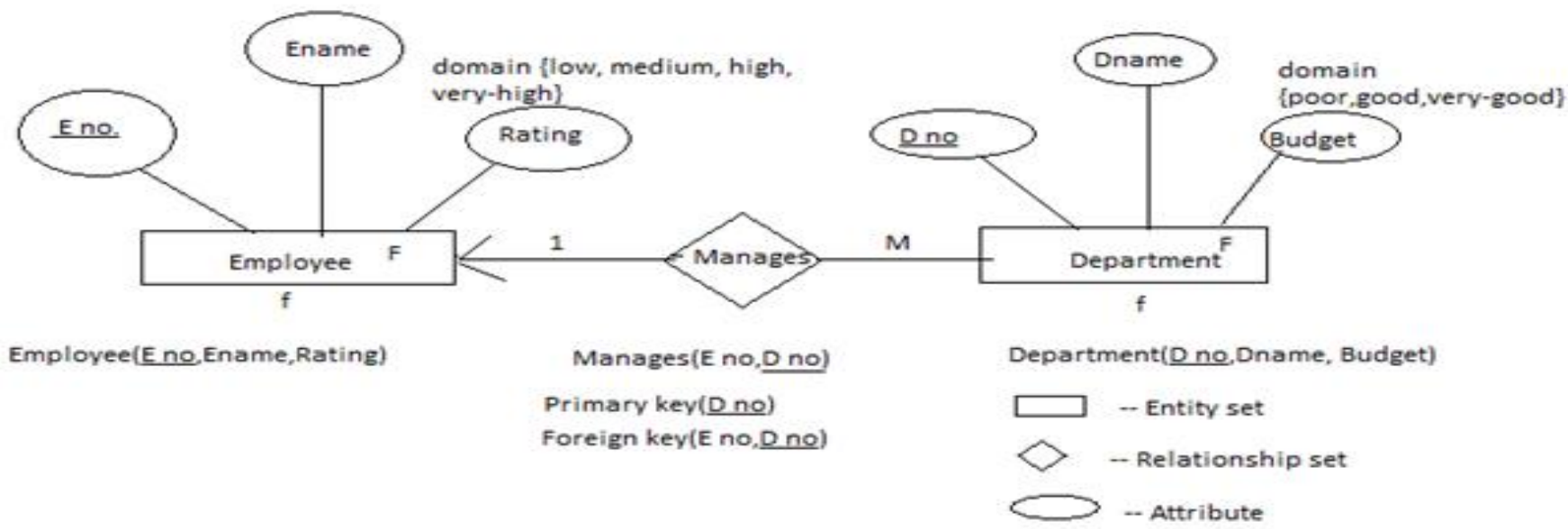

In fuzzy relational system E-R diagrams are used to represent attributes, entities and relationships which are shown in Fig2. In this example Employee and Department are two entities and Manages is the relationship set between them. Both the entities have fuzzy attributes, so F denotes these fuzzy entities having fuzzy attributes. Primary key of Employee is E no and that of Department is $\mathrm{D}$ no. The relationship between Employee and Department is 1: M (one to many), so primary key of Manages is $\underline{D}$ no. Foreign keys in Manages are E no and D no because they reference to primary keys of other constraints. Foreign key can also be a primary key.

\section{LIMITATIONS OF FOOD OVER FRDB}

- The Fuzzy object oriented database model's weakest aspect appears to be its relative lack of support for ad hoc associative access. Relational databases have capitalized in this area: SQL makes it possible to query any relational database without having to learn an entirely new language for each one. FOOD models are far from adopting any standard query language, and there exists some resistance to ad hoc querying because such activity violates the encapsulation concept. 
- The FRDB management system provided a comprehensive solution to the business database design and management needs supplying both a data model and a set of fairly straightforward normalization rules to design and evaluate relational databases, but FOOD management system does not provide such tools. [2]

\section{INTEGRATION OF RELATIONAL SQL WITH OODB}

The above problem is overcome by integration of FOOD management system and FRDB management system, which provides an extension to relational SQL. This helps in collecting related records easily with simple query .The database can make use of relationships between data. Let us consider an example to show that how integration of SQL with FOOD make query simple. [2]

SQL Query in database:

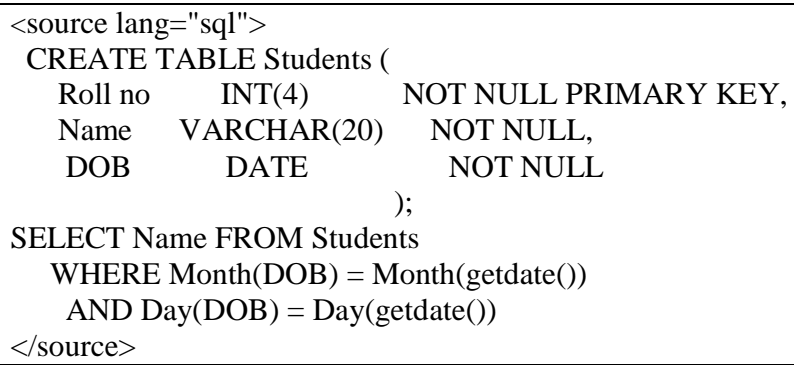

SQL databases allow crafting of custom [FUNCTION], so query would appear as below:

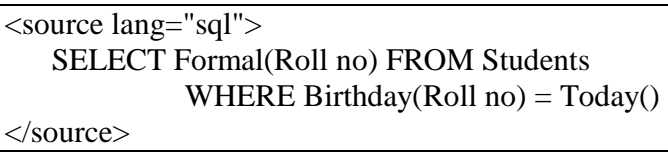

In Object relational database query would be like given below with user-defined data-types and expressions such as $<$ code $>$ BirthDay ()$</$ code $>$ :

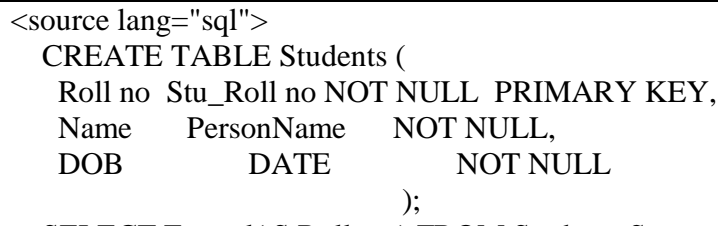

The object-relational model can offer another advantage in that the database can make use of the relationships between data to easily collect related records. But in relational database, collecting information of related records requires a "join":

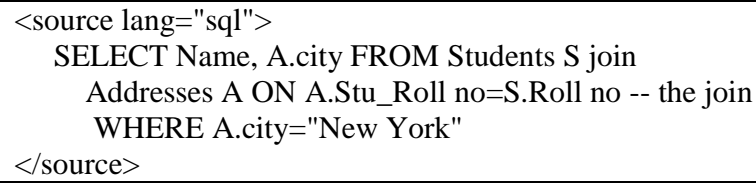

Object-relational database query is much simpler:

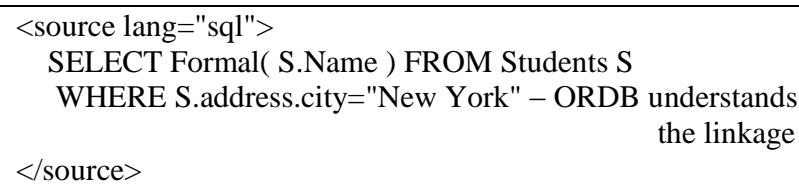

\section{CONCLUSION}

SQL-DBMS products focused on the efficient management of data drawn from a limited set of data-types. Whereas fuzzy object oriented database systems focused on abstraction, inheritance, behavior and encapsulation. Both databases have their own advantages. So to deal with more complex query, Fuzzy objectrelational database (FORDB) is developed which allow developers to raise the level of abstraction at which they view the problem domain. These database add new object storage capabilities to the relational database systems, which integrate management of traditional fielded data, complex objects such as geospatial data and time series and binary media such as images, audio, video and applets. FORDB management system server can execute data manipulation operations and complex analytical operations to search and transform multimedia and other complex objects.

\section{REFERENCES}

[1] Amel Grissa Touzi and Mohamed Ali Ben Hassine, "New Architecture of Fuzzy Database Management Systems", "the International Arab Journal of Information Technology, Vol. 6, No. 3, July 2009”.

[2] Bipin C. Desai, "An introduction to database systems" eight edition, 2012.

[3] Cristina-Maria Vladarean, "Extending Object-Oriented databases for fuzzy information modeling", ROMAI J., 2, 1 (2006), 225-237.

[4] "FRDBMS for Fuzzy Querying based on GEFRED Model" IJCA Proceedings on National Workshop-Cum-Conference on (RTMC2011), 2012.

[5] Jose Galindo, "Handbook of research on fuzzy information processing in databases", IGI Global, 2008.

[6] P.K.Shukla, M.Darbari, V. K. Singh, S.P.Tripathi, "A Survey of Fuzzy Techniques in object oriented databases", International Journal of Scientific \& Engineering Research, Volume 2, Issue 11, November-2011.

[7] R. De Caluwe, N, Van Gyseghem, V. Cross, "Basic notions and rationale of the integration of uncertainty management and object-oriented databases", 1997.

[8] S.K.Mondal, J.sen, Md.R.Islam and Md.S.Hossian, "Performance comparison of fuzzy queries on fuzzy database and classical database," International journal of Computer Application Issue 3, Volume1 (February 2013).

[9] Z.M.M ${ }_{A}^{+}$and $L_{I}$ YAN, "A literature overview of fuzzy Database Models", Journal of Information Science and Engineering 24,189-202 (2008) 